\title{
Clinical management and outcome of extreme preterm infant with respiratory distress syndrome, early onset sepsis and necrotizing enterocolitis stage 1: a case report
}

\author{
A. Priya, Mohammed Fardan, Aswathy M. Shaji, R. Kannan, K. Arun Chander Yadav*
}

Department of Clinical Pharmacology, Apollo Children's Hospital, Chennai, Tamil Nadu, India

Received: 05 March 2021

Revised: 10 April 2021

Accepted: 12 April 2021

\section{*Correspondence:}

Dr. K. Arun Chander Yadav,

Email: clinicalpharmaach_cni@apollohospitals.com

Copyright: (C) the author(s), publisher and licensee Medip Academy. This is an open-access article distributed under the terms of the Creative Commons Attribution Non-Commercial License, which permits unrestricted non-commercial use, distribution, and reproduction in any medium, provided the original work is properly cited.

\begin{abstract}
Respiratory distress syndrome, although it is common in extreme preterm infants, early and effective management will aid in better outcome. Preterm also comes with multiple co- morbidities which has to be considered and stepwise treatment is utmost important in tackling them. Here, we report a case of an extreme preterm baby who experienced respiratory distress syndrome with early onset sepsis along with necrotizing enterocolitis. Early diagnosis and management helped in the discharge of the infant in stable condition.
\end{abstract}

Keywords: Respiratory distress syndrome, Sepsis, Necrotizing enterocolitis, Preterm infant

\section{INTRODUCTION}

Respiratory distress syndrome is more common in infants born at fewer than 28 weeks gestation and affects one third of infants born at 28 to 34 weeks gestation. Immature type 2 alveolar cells produce decreased surfactant, causing a rise in alveolar surface tension and a drop in compliance. Hypoxia, cyanosis, grunting retractions, or other typical distress symptoms occur in a premature infant immediately after birth. Chest radiography is the diagnostic procedure which shows decreased lung volumes, opaque infiltrates and air bronchograms here.

In preterm infants, early onset sepsis (EOS) is most consistently defined as occurring in the first 3 days of life and is caused by bacterial pathogens transmitted from mother to infant before or during delivery. Late-onset sepsis (LOS) is evident to be occurring after 72 hours in neonatal intensive care unit (NICU) infants and 7 days of life in term infants, which has been variably defined as occurring up to the age of 90 or 120 days, and may be caused by vertically or horizontally acquired pathogens.
Antimicrobials used to treat sepsis in neonates usually comprises of beta-lactams such as ampicillin, oxacillin, and cefotaxime; extended-spectrum beta-lactams such as piperacillin-tazobactam and the carbapenem (meropenem). ${ }^{1}$

Necrotizing enterocolitis (NEC) is the most common life threatening gastrointestinal emergency which cause's damage to the immature gastrointestinal mucosa and results in loss of mucosal integrity. ${ }^{2}$ The initiation of enteral feeding opens up the stage for bacterial proliferation and damaged mucosa is invaded by gas producing bacteria. Antenatal steroids, human milk feedings, adoption of standardized feeding regimen, and probiotics are used for the prevention of NEC.

Neonatal bacterial meningitis is a devastating infection. It is the acute inflammation of the meninges, subarachnoid space, and brain vasculature resulting from infection. To confirm the diagnosis, determination of the causative pathogen and refining antimicrobial therapy by the collection of cerebrospinal fluid (CSF) by performing an 
LP is essential. Empiric antibiotics used in suspected meningitis require adequate CSF penetration and sensitivity against the pathogen.

Ventricular septal defect (VSD) is a heart defect most often present at birth involves a hole in the wall between the lower chambers of the heart. The incidence of congenital heart disease is higher in the low birth weight group and preterm babies. ${ }^{3}$ Most holes close on their own, although many may need surgery to close the hole.

Hydrocephalus is one of the most common congenital abnormalities affecting the nervous system. It results from hindrance of CSF pathways by a varied range of developmental, genetic, and acquired abnormalities and can have negative consequences on the neuro developmental outcome of affected neonates. The surgical insertion of a drainage system, called a shunt is the most usual treatment for hydrocephalus.

\section{CASE REPORT}

An extreme preterm baby of 28 week 3 day of gestation was admitted in NICU after emergency lower segment caesarean section (LSCS). The mother had maternal complications like pregnancy induced hypertension, gestational diabetes mellitus, and uncontrolled hypothyroidism and admitted 3 days back with high blood pressure (BP), proteinuria suggestive of severe preeclampsia. She had received two doses of steroids 36 hours before delivery and started on magnesium sulphate infusion. The birth weight was recorded to be 640 gram. Baby was started on conventional mode of ventilation. Chest $\mathrm{X}$ ray and arterial blood gas (ABG) done at birth was normal and ventilator settings were adjusted. She required inotrope support with dobutamine $5 \mathrm{mcg} / \mathrm{kg} / \mathrm{min}$ for 10 hours to maintain mean arterial blood pressure.

On day 3 of life, echo was done which showed large pPatent ductus arteriosus (PDA) with sub aortic VSD and advised to start on intravenous (IV) frusemide and oral aldactone. She was given IV paracetamol for 5 days and repeat echo showed closed PDA with patent foramen ovale (PFO) and sub aortic VSD. In view of feed intolerance, acute kidney injury (AKI) and persistent hyponatremia, diuretics were withheld. On day 10 of life she was extubated to high flow nasal oxygen support (HFNC).

On day 58, baby had setback inform of apnea and desaturation for which baby was intubated and connected to mechanical ventilator. Chest $\mathrm{X}$ ray showed pulmonary markings and repeat echo showed moderate sized sub aortic VSD, increased left ventricular volume overload to restart intravenous furosemide and aldactone. In view of extreme prematurity, baby was started on injection piptaz along with prophylactic fluconazole after sending partial septic screening. Initial counts showed normal count with mild thrombocytopenia (1.1 lakh) and C-reactive protein (CRP) was positive (38). On day 2 of life, baby developed abdominal distension with ultrasound abdomen showed features of NEC stage 1. Hence antibiotics were escalated to inj meropenam and inj metronidazole on day 2 of life. Repeated CRP had reducing trends (13) and abdominal distension started to improve. Blood culture at the end of day 5 was sterile; hence antibiotics were de-escalated to piptaz. Repeated CRP at 13 day of life was negative, hence piptaz was stopped after 7 days of antibiotic.

On day 32 of life, baby developed respiratory distress and desaturation; hence blood culture was sent and started on IV meropenem. Clinically, baby was lethargic and had bulging AF and hypotonia of all 4 limbs. CSF analysis was done and reports were suggestive of pyogenic meningitis (turbid cell count: $2100 \mathrm{~N}$ : 93 L: 7). Blood culture grew Enterobacter colacea, for which infectious team was involved. After 14 days of antibiotic she was continued to be lethargic, hence fungal sepsis was suspected and fluconazole was escalated to amphotericin B. Repeated blood culture was sterile and CSF analysis on day 57 of life showed improvement in cell counts and culture was sterile.

She noted to develop enlarging head circumference and sunset sign suggestive hydrocephalus. Functional neurosonogram showed increased ventricle size and neurosurgery review was obtained and suggested conservative management. She received meropenem for total period of 28 days, cefotaxime for 7 days, amphotericin B for 14 days, and vancomycin for 7 days. She developed persistent increase in head circumference. Neurosonogram was done which showed prominent ventricular system (both lateral ventricles) with moderate turbidity suggested obstructive hydrocephalus. Neurosurgeon opinion obtained and serial anterior fontanelle tapping was done weekly once for thrice. Size of head circumference was being static, so advice to follow with neurosurgeon.

\section{DISCUSSION}

In NICU, respiratory management of extremely preterm infants occurs in the form of conservative or invasive, where by infants are frequently intubated and placed on mechanical ventilation during acute phase i.e. 72 hours after birth. In all countries including Japan this approach of intubating and placing infants on mechanical ventilation emerged as the most common respiratory management strategy used for infants born at 23-24 weeks of gestation. ${ }^{4}$ In this case, baby was continued on mechanical ventilation with minimal setting.

C-reactive protein, procalcitonin, and hematologic scoring system are used in the diagnosis of infants with suspected sepsis. Beta-lactams such as ampicillin, oxacillin and cefotaxime; extended spectrum betalactams such as piperacillin-tazobactam, carbapenam and meropenem are the antimicrobials used to treat sepsis in neonates, it act by inhibiting the peptidoglycan layer of bacterial cell wall. ${ }^{5}$ In this case injection piptaz and prophylactic fluconazole was started in view of CRP which was positive. 
Evidence over the past 13 years suggests that fluconazole prophylaxis for the prevention of invasive candidiasis is safe and effective in premature infants. ${ }^{6}$ Medical management followed for NEC was no oral feeding, gastric suction, parenteral nutrition, intravenous broad spectrum antibiotic therapy, cardio respiratory support, followed up by serial estimation of blood gases, platelet count, complete blood count, and serial abdominal $\mathrm{X}$ ray studies. ${ }^{7}$ Here in this case, in view of extreme prematurity and abdominal Doppler finding, baby was kept in nil by mouth and started on parenteral nutrition. After the confirmation of NEC by ultrasound, baby was continued to be in NPO and started on injection meropenem and metronidazole for 5 days.

South Australian neonatal medication guidelines suggest the use of intravenous metronidazole for infection due to susceptible anaerobic organisms. ${ }^{8}$ Here the baby had NEC, so metronidazole was given for 7 days against the susceptible anaerobic microorganisms.

Among the 3910 newborns admitted over 10 year study period, 24 presented with meningitis and that was confirmed by positive CSF culture. Common procedures were parenteral nutrition, mechanical ventilation and previous antibiotic therapy. CRP level was significantly higher in cases of meningitis caused by gram negative agents. The clinical course of gram negative meningitis was worse and a large number of patients developing ventriculitis and hydrocephaly. ${ }^{9}$ In this case CSF analysis done and reports were suggestive of pyogenic meningitis. Blood culture grew Enterobacter Cloacae, a gram negative anaerobic bacterium, therefore continued on IV meropenem.

Invasive candidiasis is a common fungal infection seen in premature neonates. Neonates with low birth weight have an increased risk of fungal infection due to their immature immune system, fragile skin and mucosal barriers as well as under developed bacterial flora. ${ }^{10}$ Since, the baby was lethargic, after 14 days of meropenem, fungal sepsis was suspected and fluconazole was escalated to amphotericin $\mathrm{B}$ and given for 14 days.

The introduction of cefotaxime provided an alternative option for the treatment for meningitis caused by gram negative bacterium. This is based on the lower minimum bactericidal concentration (MBCs) of gram negative bacteria to cefotaxime compared to penicillins and aminoglycosides with high CSF concentration that can be safely achieved. Cefotaxime increases the proportion of infants who will have sterile CSF cultures 48-72 hours into treatment. ${ }^{11}$ In this case, cefotaxime was given for 7 days to treat the same.

The basic principle of hydrocephalus treatment is bypassing the site of obstruction to CSF flow by diverting the CSF from ventricular cavity to a site where it is readily absorbed. On the basis of this principle, shunt procedure have become the mainstay of treatment in severe hydrocephalus. ${ }^{12}$

Here, anterior fontanelle tapping was done weekly once or thrice. Size of head circumference was remaining static, so was advised to be followed under the guidance of neurosurgeon.

Vancomycin and third generation cephalosporin e.g. cefotaxime should be considered for late onset sepsis in a neonate presenting with cardio-respiratory instability and in areas where methicillin-resistant Staphylococcus aureus (MRSA) is prevalent. $^{13}$ Here vancomycin too was considered and administered for 7 days prior to taking repeat culture test.

Baby was finally discharged after spending three months of her life in NICU with a weight of $1.02 \mathrm{~kg}$, active vitals, normothermic and with a regular follow up plan.

\section{CONCLUSION}

Earlier screening for probable ill-conditioning of the neonate is utmost necessary in extreme preterm infants. Earlier medical management will help in effective therapeutic outcome which if delayed would lead to dire consequences in condition. Here a case of an extreme preterm baby was presented, who experienced respiratory distress syndrome with early onset sepsis along with necrotizing enterocolitis and stepwise treatment approach lead to successful discharge of patient.

\section{ACKNOWLEDGEMENTS}

Authors would like to thank Department of Neonatology of Apollo Children's Hospital to allow them to go through patient's data thoroughly.

Funding: No funding sources

Conflict of interest: None declared

Ethical approval: Not required

\section{REFERENCES}

1. Thakuria B, Lahon K. The Beta Lactam Antibiotics as an Empirical Therapy in a Developing Country: An Update on Their Current Status and Recommendations to Counter the Resistance against Them. J Clin Diagn Res. 2013;7(6):1207-14.

2. Gephart SM, McGrath JM, Effken JA, Halpern MD. Necrotizing Enterocolitis Risk. Adv Neonatal Care. 2012;12(2):77-89.

3. Kramer HH, Trampisch HJ, Rammos S, Giese A. Birth weight of children with congenital heart disease. Eur $\mathbf{J}$ Pediatr. 1990;149(11):752-7.

4. Isayama T. The clinical management and outcomes of extremely preterm infants in Japan, past, present and future. Transl Pediatr. 2019;8(3):199-211. 
5. Simonsen KA, Anderson-Berry AL, Delair SF, Davies HD. Early onset neonatal sepsis. Clin Microbiol Rev. 2014;27(1):21-47.

6. Ericson JE, Benjamin DK. Fluconazole prophylaxis for prevention of invasive candidiasis in infants. Curr Opin Pediatr. 2014;26(2):151-6.

7. Abdel Maksod YH, Shahin AM, Ahmed H, Aabdelwahab MB. Stepwise management of necrotizing enterocolitis could improve outcome of this life- Threatening disease. Med Rep Case Stud. 2016;1:2.

8. South Australian Neonatal medication guidelines, government of South Australia. South Australian Maternal, Neonatal and Gynaecology community of practice. 2017;1-3.

9. Bentlin MR, Ferreira GL, de Souza Rugolo LMS, Silva GHS, Mondelli AL, et al. Neonatal meningitis according to the microbiological diagnosis. Arq Neuropsiquiatr. 2010;68(6):882-7.

10. Schellack N. Amphotericin B in the management of fungal infections in a neonatal intensive care unit; experiences in a teaching hospital. South Afr J Epidemiol Infect. 2012;27(1).

11. Heath PT, Yusoff NKK, Baker CJ. Neonatal meningitis. Arch Dis Child Fetal Neonatal. 2003;88:73-8.

12. Venkataramana NK. Hydrocephalus Indian scenarioA review. J Pediatr Neurosci. 2011;6(1):511-22.

13. Sivanandan S, Soraisham AS, Swarnam K. Choice and duration of antimicrobial therapy for neonatal sepsis and meningitis. Int J Pediatr. 2011;1-9.

Cite this article as: Priya A, Fardan M, Shaji AM, Kannan R, Yadav KAC. Clinical management and outcome of extreme preterm infant with respiratory distress syndrome, early onset sepsis and necrotizing enterocolitis stage 1: a case report. Int J Basic Clin Pharmacol 2021;10:580-3. 